\title{
Regioselective synthesis of plant (iso)flavone glycosides in Escherichia coli
}

\author{
Xian-Zhi He • Wen-Sheng Li • Jack W. Blount • \\ Richard A. Dixon
}

Received: 30 July 2007 / Revised: 20 May 2008 / Accepted: 21 May 2008 / Published online: 21 June 2008

(C) The Author(s) 2008

\begin{abstract}
The flavonoids genistein, biochanin A, luteolin, quercetin, and kaempferol are plant natural products with potentially useful pharmacological and nutraceutical activities. These natural products usually exist in plants as glycosides, and their glycosylation has a remarkable influence on their pharmacokinetic properties. The glycosyltransferases UGT71G1 and UGT73C8 from Medicago truncatula are excellent reagents for the regioselective glycosylation of (iso) flavonoids in Escherichia coli grown in Terrific broth. Ten to $20 \mathrm{mg} / \mathrm{L}$ of either genistein or biochanin A 7-O-glucoside was produced after feeding genistein or biochanin A to $E$. coli expressing UGT71G1, and similar levels of luteolin 4'-O- and 7-O-glucosides were produced after feeding luteolin to cultures expressing UGT73C 8 . For the production of kaempferol 3-O-glucoside or quercetin 3-O-glucoside, the Phe148Val or Tyr202Ala mutants of UGT71G1 were employed. Ten to $16 \mathrm{mg} / \mathrm{L}$ of either kaempferol 3-O- or quercetin 3-O-glucosides were produced on feeding kaempferol or quercetin to $E$. coli expressing these enzymes. More than $90 \%$ of the glucoside products were released to the medium, facilitating their isolation.
\end{abstract}

\section{Introduction}

Polyphenolic flavonoids are common constituents of vegetables, fruits, and herbal medicines. Their health-promoting

Electronic supplementary material The online version of this article (doi:10.1007/s00253-008-1554-7) contains supplementary material, which is available to authorized users.

X.-Z. He $(\varangle) \cdot$ W.-S. Li $\cdot$ J. W. Blount $\cdot$ R. A. Dixon

Plant Biology Division, The Samuel Roberts Noble Foundation,

2510 Sam Noble Parkway,

Ardmore, OK 73401, USA

e-mail: xianzhihe@noble.org effects have long been recognized, and flavonoids such as genistein, biochanin A, quercetin, kaempferol, and luteolin have been exploited for their medicinal and nutritional activities (Arai et al. 2000; Erdman et al. 2007; Mink et al. 2007). The isoflavone genistein, biochanin A, and the flavonols quercetin and kaempferol (Fig. 1) have been shown to reduce the incidence of cancer (Caltagirone et al. 2000; Kao et al. 1998; Kellis and Vickerry 1984; Shenouda et al. 2004; Way et al. 2004) and cardiovascular disease (Arai et al. 2000; Cogolludo et al. 2007; Hertog et al. 1993; Mink et al. 2007) and also to exhibit anti-inflammatory activities (Gabor 1979; Kumazawa et al. 2006; Lewis 1989). Many studies have been aimed at developing new production platforms for such plant natural products through metabolic engineering in plants or microorganisms (Deavours and Dixon 2005; Leonard et al. 2005, 2006; Lim et al. 2004; Liu et al. 2002; Willits et al. 2004; Yu et al. 2000, 2003).

Most phenolic secondary metabolites exist in plants as glycosides, often with more than one hydroxyl group glycosylated. Metabolic engineering by expression of isoflavone synthase in Arabidopsis, tobacco, soybean, and alfalfa leads to accumulation of genistein glycosides rather than the aglycone (Deavours and Dixon 2005; Liu et al. 2002; Yu et al. 2000, 2003). Glycosylation of the aglycones increases their solubility and stability (Hollam et al. 1995, 1996, 1999; Smith et al. 2000) and also increases their bioavailability in mammals (Crespy et al. 2001; Graefe et al. 2001; Hollman and Katan 1998, 1999). Furthermore, the position of glycosylation, for example 4'-O-, 3-O-, or 3-, 4'-di- $O$-glucosides in the case of quercetin, significantly impacts hydrolysis and bioavailability (Cermak et al. 2004; Day et al. 2003; Gee et al. 2000). For example, quercetin 3-O- and 4'-O-glucosides, but not the 3-,4'-O-diglucoside, were transported through the rat small intestine, and during passage across the epithelium the monoglucoside was 

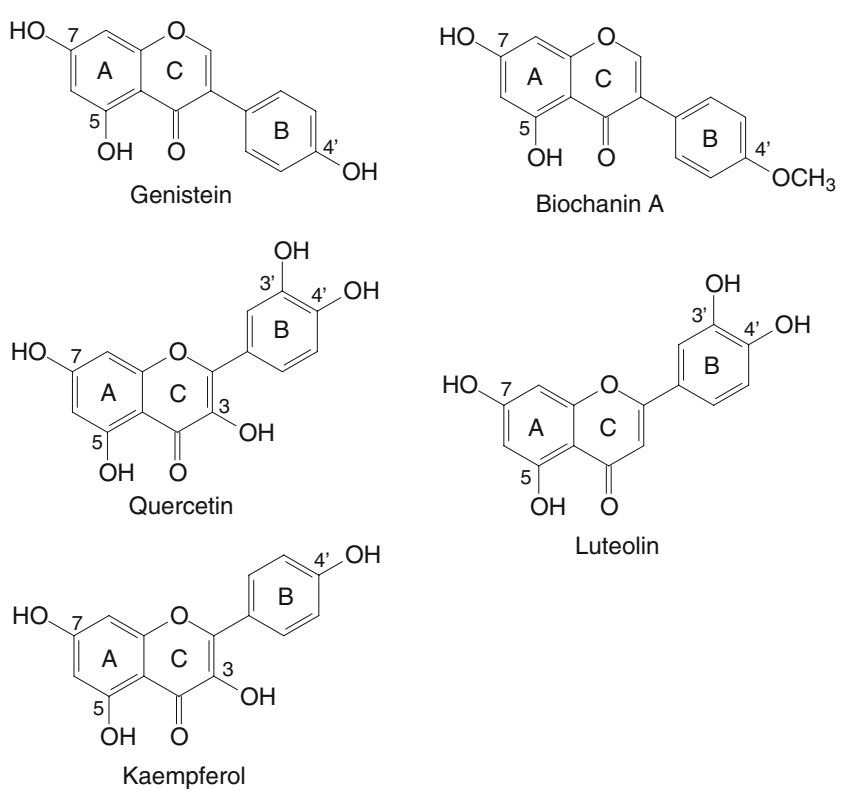

Fig. 1 Chemical structures of the isoflavones genistein and biochanin $\mathrm{A}$, the flavone luteolin, and the flavonols quercetin and kaempferol

rapidly deglycosylated and then glucuronidated into quercetin 3-O- and 7-O-glucuronide (Gee et al. 2000).

Availability of a range of (iso)flavonoids with different regiospecific glycosyl substituents will facilitate research on their biological utilization. However, regiospecific chemical synthesis of polyphenolic glycosides is far from trivial (Bouktaib et al. 2002; Li et al. 2002). We have previously characterized the glycosyltransferases UGT71G1 and UGT73C8 from the model legume Medicago truncatula (Achnine et al. 2005; Modolo et al. 2007; Shao et al. 2005). UGT71G1, a member of group E family 1 glycosyltransferases, regioselectively glycosylates the 7-O position of genistein and biochanin $\mathrm{A}$ but produces five different monoglucosides from quercetin, with the 3'-O-glucoside predominating (Achnine et al. 2005; He et al. 2006). The structure of UGT71G1 has been determined and belongs to the GT-B fold with Rossmann-like domains (Shao et al. 2005). Two structure-directed mutants of the enzyme, Phe148Val and Tyr202Ala, predominantly glycosylated the 3-O position of quercetin in vitro (He et al. 2006). UGT73C8, closely related to UGT72C1 (72\% identity) in group D of family 1 glycosyltransferases, showed enzyme activity preference for isoflavonoids but also exhibited high activity with the flavone luteolin (Modolo et al. 2007). However, the regioselectivity and effectiveness of these enzymes as biocatalysts for the synthesis of (iso) flavonoid glycosides in vivo remains to be evaluated. Here, we report the production of milligram quantities of the 7-O-glucosides of genistein and biochanin $\mathrm{A}$, the 4'-Oand 7-O-glucosides of luteolin, and the 3-O-glucosides of quercetin and kaempferol in Escherichia coli cultures expressing UGT71G1, UGT73C8, and mutants of UGT71G1, respectively.

\section{Materials and methods}

Bacterial growth, induction, precursor feeding, and product extraction

General chemicals and quercetin 3-O-glucoside were purchased from Sigma-Aldrich (St. Louis, MO, USA). All other (iso) flavones and their glycosides were purchased from Indofine Chemicals (Hillsborough, NJ, USA). Kaempferol 4'-O-glucoside was purchased from Extrasynthese (Genay, France). The protein expression vector pET28a and E. coli strain BL21(DE3) were from Novagen (Madison, WI, USA).

The glycosyltransferases UGT71G1, UGT73C8, and UGT71G1 mutants Phe148Val and Tyr202Ala were cloned into pET 28a as described previously (He et al. 2006). E. coli BL21 (DE3) strains carrying these constructs were grown in Terrific broth (TB) or Luria-Bertani (LB) medium at $37^{\circ} \mathrm{C}$ until $\mathrm{OD}_{600}$ reached 0.7 . Isopropyl-1-thio- $\beta$-Dgalactopyranoside was then added to the cultures to a final concentration of $0.5 \mathrm{mM}$, and the cultures were incubated at $20^{\circ} \mathrm{C}$ for $5 \mathrm{~h}$. Substrate (genistein, biochanin A, luteolin, quercetin, or kaempferol) dissolved in dimethyl sulfoxide (DMSO) was then added to the cultures to a final concentration of 50 or $100 \mu \mathrm{M}$. E. coli cultures harboring empty vector pET $28 \mathrm{a}$ vector were used as a control. Cells were harvested at different time points after addition of substrates. The medium and bacterial cells were separated by centrifugation for $30 \mathrm{~min}$ at $4,000 \times g$ at $4^{\circ} \mathrm{C}$. The medium was acidified with $6 \mathrm{~N} \mathrm{HCl}$ to $\mathrm{pH} 1$, extracted twice with ethyl acetate, and dried under nitrogen gas. Residues were resuspended in methanol for high-performance liquid chromatography (HPLC) analysis. The cell pellet was resuspended in $\mathrm{MeOH}-\mathrm{HCl}(v / v, 10: 1)$ and extracted for $1 \mathrm{~h}$ in an ultrasonic bath as described previously (Willits et al. 2004). Insoluble cell debris was removed by centrifugation and the extract was dried under nitrogen gas. Residues were resuspended in methanol for HPLC analysis. For large-scale cultures, $500 \mathrm{~mL}$ of bacterial culture were grown in a 2-L flask in TB medium. Bacterial induction was as described above. Twenty-four hours after the addition of substrate (100- $\mu \mathrm{M}$ final concentration), cells were harvested and products were extracted and analyzed as described above.

Soluble protein purification

UGT71G1, UGT71G1 mutants F148V and Y202A, and UGT73C8 soluble proteins were purified from induced cell 
cultures using the MagneHis protein purification system according to the manufacturer's instructions (Promega, Madison, WI, USA). Protein concentration was determined with the Bio-Rad protein dye-binding assay (Bio-Rad Laboratories Inc., Hercules, CA, USA) using bovine serum albumin as standard.

\section{HPLC analysis of glycosylated products}

Reverse-phase HPLC analysis was carried out on a Hewlett Packard 1100 system using a 5 - $\mu \mathrm{m}$ C18 column $(250 \times$ $4.6 \mathrm{~mm}$, Waters spheroisorb $5 \mu \mathrm{m}$ ODS2) with the following gradient: $\mathrm{A}=1 \%$ phosphoric acid, $\mathrm{B}=$ acetonitrile, $0-5 \mathrm{~min}$, 5\% B; 5-10 min, 10\% B; 10-25 min, 17\% B; 25-30 min, 23\% B, 30-65 min, 50\% B; 65-79 min, 100\% B; 79-80 min, 5\% B. The eluate was monitored at $254 \mathrm{~nm}$. The glycosylated products were identified according to their retention times, UV spectra, and comparison to authentic standards (Lim et al. 2004).

\section{Results}

Production of genistein and biochanin A 7-O-glucoside in $E$. coli

UGT71G1 is a multifunctional triterpene-flavonoid glycosyltransferase with specific activity for the triterpene medicagenic acid, the isoflavones genistein and biochanin A, and the flavonol quercetin. Recombinant $M$. truncatula UGT71G1 can transfer glucose from uridine diphosphate (UDP)-glucose to genistein or biochanin $\mathrm{A}$ to produce genistein or biochanin A 7-O-glucoside in vitro (Achnine et al. 2005; He et al. 2006; Shao et al. 2005). To investigate the production of genistein or biochanin A 7-O-glucosides in $E$. coli in vivo, we incubated induced cell cultures of $E$. coli BL21(DE3) carrying the UGT71G1-pET 28a construct with different concentrations of genistein or biochanin $\mathrm{A}$ for different times. The medium and cell pellet were extracted with ethyl acetate, and products were analyzed by HPLC. The expected 7-O-glucosides were produced under all conditions used and could be detected in the culture medium after $5 \mathrm{~h}$ (Fig. 2, Table 1). More than $70 \%$ of genistein substrate was converted to the 7-O-glucoside by the $E$. coli cultures after $24-\mathrm{h}$ incubation. The product yield was about $20-\mathrm{mg} / \mathrm{L}$ TB culture medium after 24-h incubation with $100 \mu \mathrm{M}(27.02 \mathrm{mg} / \mathrm{L})$ genistein. In comparison to LB culture medium, a 3.5-fold higher 7-O-glucoside yield was achieved in TB culture medium, with twice as much glucoside produced with $100 \mu \mathrm{M}$ as compared to $50 \mu \mathrm{M}$ genistein after 24-h incubation (Fig. 2, Table 1). A similar glucoside production pattern was observed when the culture was incubated with biochanin A. Thirteen milligram per liter biochanin A 7-O-glucoside was produced after 24-h incubation with $100 \mu \mathrm{M}(28.42 \mathrm{mg} / \mathrm{L})$ biochanin A substrate in TB medium. About threefold higher levels of 7-O-glucoside were produced in TB medium compared to LB medium (Fig. S1, Table 1). No genistein or biochanin A 7-Oglucoside was detected in the medium from $E$. coli harboring the vector control (Figs. S2 and S3).

To evaluate production efficiency on a larger scale, we set up 500-mL TB medium cultures, expressing the corresponding glycosyltransferases or the mutants described above, in 2-L flasks. The substrates were incubated individually for $24 \mathrm{~h}$ in the cell cultures and the glucoside products were analyzed by HPLC. The glucoside product yield was about $10-16-\mathrm{mg} / \mathrm{L}$ culture medium with conversion rates from $30 \%$ to $60 \%$ (Table 2 ). The efficiency was about $80 \%$ to that of the small-batch scale. In both cases, more than $90 \%$ of the glycosylated products were released to the medium.

To evaluate the effects of the (iso)flavonoid substrates on the growth of E. coli, we monitored bacterial growth at
Fig. 2 Production of genistein 7-O-glucoside using UGT71G1 as a biocatalyst in $E$. coli. Time course showing levels of genistein 7-O-glucoside in culture medium of engineered $E$. coli expressing UGT71G1 fed with genistein as substrate (squares $=$ 7-O-glucoside level in TB medium, diamonds $=7-O$-glucoside level in LB medium). Insert showed the levels of aglycone consumed during the time course (triangles $=$ aglycone level in $\mathrm{TB}$ medium, circles $=$ aglycone level in LB medium). Fifty micromolar (13.51-mg/L final concentration) genistein added to the bacterial culture

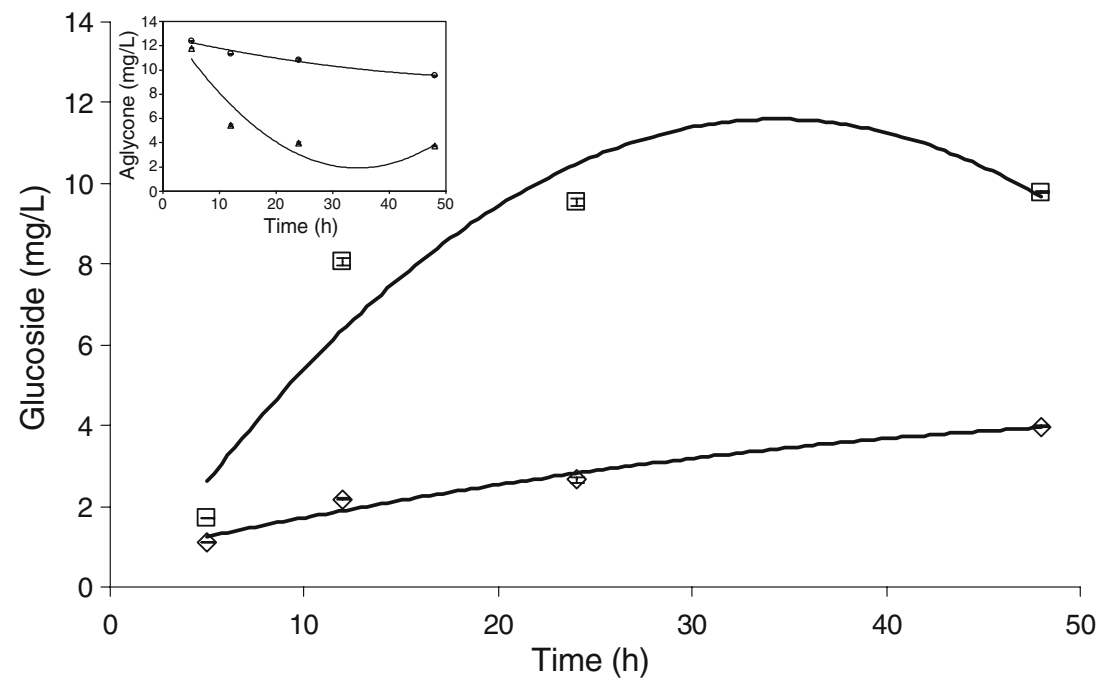


Table 1 (Iso)flavone glucoside synthesis in E. coli in LB and TB culture media (100 $\mu \mathrm{M}$ substrate)

\begin{tabular}{|c|c|c|c|c|c|c|c|c|c|}
\hline \multirow[b]{2}{*}{ Construct } & \multirow[b]{2}{*}{ Glucoside } & \multicolumn{4}{|c|}{ LB medium (mg/L) } & \multicolumn{4}{|c|}{ TB medium $(\mathrm{mg} / \mathrm{L})$} \\
\hline & & $5 \mathrm{~h}$ & $12 \mathrm{~h}$ & 24 & $48 \mathrm{~h}$ & $5 \mathrm{~h}$ & $12 \mathrm{~h}$ & $24 \mathrm{~h}$ & $48 \mathrm{~h}$ \\
\hline $\begin{array}{l}\text { UGT71G1 wild } \\
\text { type }\end{array}$ & $\begin{array}{l}\text { Genistein 7-O- } \\
\text { tglucoside }\end{array}$ & $2.05 \pm 0.02$ & $4.39 \pm 0.14$ & $5.57 \pm 0.07$ & $5.58 \pm 0.13$ & $3.95 \pm 0.03$ & $15.45 \pm 0.28$ & $20.06 \pm 0.16$ & $17.90 \pm 0.61$ \\
\hline $\begin{array}{l}\text { UGT71G1 wild } \\
\text { type }\end{array}$ & $\begin{array}{l}\text { Biochanin A 7-O- } \\
\text { tglucoside }\end{array}$ & $2.08 \pm 0.03$ & $3.77 \pm 0.16$ & $4.05 \pm 0.33$ & $4.16 \pm 0.10$ & $2.19 \pm 0.09$ & $9.50 \pm 0.24$ & $13.02 \pm 0.57$ & $13.18 \pm 0.14$ \\
\hline $\begin{array}{l}\text { UGT71G1 mutant } \\
\text { F148V }\end{array}$ & $\begin{array}{l}\text { Quercetin 3-O- } \\
\text { tglucoside }\end{array}$ & $2.94 \pm 0.06$ & $6.55 \pm 0.64$ & $8.20 \pm 0.32$ & $5.95 \pm 0.55$ & $2.00 \pm 0.08$ & $5.93 \pm 0.22$ & $11.70 \pm 0.03$ & $16.75 \pm 0.65$ \\
\hline $\begin{array}{l}\text { UGT71G1 mutant } \\
\text { Y202A }\end{array}$ & $\begin{array}{l}\text { Quercetin 3-O- } \\
\text { tglucoside }\end{array}$ & $4.15 \pm 0.37$ & $9.84 \pm 0.21$ & $10.09 \pm 0.22$ & $7.86 \pm 0.87$ & $1.81 \pm 0.08$ & $6.84 \pm 0.12$ & $12.37 \pm 0.06$ & $19.88 \pm 1.26$ \\
\hline $\begin{array}{l}\text { UGT71G1 mutant } \\
\text { F148V }\end{array}$ & $\begin{array}{l}\text { Kaempferol 3-O- } \\
\text { tglucoside }\end{array}$ & $1.05 \pm 0.07$ & $3.56 \pm 0.04$ & $3.92 \pm 0.07$ & $5.06 \pm 0.02$ & $1.71 \pm 0.04$ & $10.59 \pm 0.35$ & $15.81 \pm 0.40$ & $14.83 \pm 0.19$ \\
\hline $\begin{array}{l}\text { UGT71G1 mutant } \\
\text { Y202A }\end{array}$ & $\begin{array}{l}\text { Kaempferol 3-O- } \\
\text { tglucoside }\end{array}$ & $1.18 \pm 0.03$ & $3.82 \pm 0.07$ & $7.14 \pm 0.09$ & $7.81 \pm 0.16$ & $2.10 \pm 0.08$ & $12.40 \pm 0.14$ & $16.46 \pm 0.46$ & $18.59 \pm 0.41$ \\
\hline $\begin{array}{l}\text { UGT73C8 wild } \\
\text { type }\end{array}$ & $\begin{array}{l}\text { Luteolin 4'-O- } \\
\text { tglucoside }\end{array}$ & $1.56 \pm 0.11$ & $8.45 \pm 0.38$ & $9.82 \pm 0.24$ & $13.68 \pm 0.19$ & $2.78 \pm 0.04$ & $11.78 \pm 0.06$ & $14.01 \pm 0.44$ & $15.49 \pm 0.34$ \\
\hline $\begin{array}{l}\text { UGT73C } 8 \text { wild } \\
\text { type }\end{array}$ & $\begin{array}{l}\text { Luteolin 7-O- } \\
\text { tglucoside }\end{array}$ & $1.00 \pm 0.02$ & $4.76 \pm 0.04$ & $4.96 \pm 0.03$ & $7.95 \pm 0.07$ & $1.26 \pm 0.01$ & $7.02 \pm 0.05$ & $8.04 \pm 0.15$ & $8.91 \pm 0.21$ \\
\hline
\end{tabular}

each time point during the culture process. There was no effect of substrates on bacterial growth. However, TB medium supported higher bacterial growth than LB medium. An example of these results with genistein is shown in Fig. 3. Soluble cellular recombinant UGT protein was also purified from the induced cell cultures grown with or without substrate to evaluate the effect of substrate on protein levels. Results showed that substrate addition did not have any significant effect on protein expression level, with $0.4-0.59$ and $0.13-0.21 \mu \mathrm{g} / \mu \mathrm{l}$ soluble recombinant protein being obtained in TB and LB medium, respectively, irrespective of substrate addition (Fig. S4, Table 3). UGT71G1 mutant F148V produced similar amounts of soluble protein in both media (data not shown).

Production of luteolin 4'-O- and 7-O-glucosides

Numerous studies have shown that luteolin [5,7,3'4'tetrahydroxy flavone (Fig. 1)] has potent anti-inflammatory properties both in vitro and in vivo (Backhouse et al. 2007; Chen et al. 2007; Karrasch et al. 2007; Kim and Jobin 2005). Luteolin inhibits the expression and production of inflammatory associated genes and mediators such as cyclooxygenase-2, tumor necrosis factor-alpha, and interleukin-6, suggesting its possible therapeutic application for treating inflammatory disorder (Chen et al. 2007). UGT73C8 can glycosylate luteolin to produce the corresponding 4'- $\mathrm{O}$ - and 7- $\mathrm{O}$-monoglucosides in vitro (Modolo et al. 2007). When luteolin (100 $\mu \mathrm{M}$, equal to $28.64 \mathrm{mg} / \mathrm{L})$ was incubated for $24 \mathrm{~h}$ with $E$. coli expressing UGT73C8 and grown in TB medium, about $14 \mathrm{mg} / \mathrm{L} 4^{\prime}-O$ glucoside and $8 \mathrm{mg} / \mathrm{L}$ 7-O-glucoside, respectively, was recovered from the medium (Fig. S5, Table 1). Similar product yield was obtained when the culture was incubated for $48 \mathrm{~h}$. The total glucoside conversion rate was about $75 \%$ in small-batch culture and $60 \%$ in the $500-\mathrm{mL}$ culture (Table 2). About 1.5-fold higher product yield was obtained in TB medium compared to LB medium. No luteolin

Table 2 (Iso)flavone glucoside synthesis in E. coli in 500-mL TB culture medium

\begin{tabular}{llcc}
\hline Construct & Glucoside & $(\mathrm{mg} / \mathrm{L})$ & Conversion rate $(\%)$ \\
\hline UGT71G1 wild type & Genistein 7- $O$-glucoside & $16.39 \pm 0.30$ & 60.7 \\
UGT71G1 wild type & Biochanin A 7- $O$-glucoside & $11.70 \pm 0.35$ & 41.2 \\
UGT71G1 mutant F148V & Quercetin 3- $O$-glucoside & $10.08 \pm 0.10$ & 29.8 \\
UGT71G1 mutant Y202A & Quercetin 3- $O$-glucoside & $11.54 \pm 0.34$ & 34.2 \\
UGT71G1 mutant F148V & Kaempferol 3- $O$-glucoside & $12.65 \pm 0.57$ & 44.2 \\
UGT71G1 mutant Y202A & Kaempferol 3- $O$-glucoside & $13.56 \pm 0.37$ & 47.4 \\
UGT73C8 wild type & Luteolin 4'- $O$-glucoside & $10.86 \pm 0.08$ & 37.9 \\
& Luteolin 7- $O$-glucoside & $6.52 \pm 0.13$ & 22.8 \\
\hline
\end{tabular}

The substrates (100- $\mu \mathrm{M}$ final concentration) were incubated in the cell culture for $24 \mathrm{~h}$. 
Fig. 3 Growth of E. coli BL21

(DE3) carrying plasmid pET28a-UGT71G1 in TB medium with $50 \mu \mathrm{M}$ genistein (filled diamonds), 100 $\mu \mathrm{M}$ genistein (filled squares), and no substrate added (filled triangles), and in LB medium with $50 \mu \mathrm{M}$ genistein (cross marks), $100 \mu \mathrm{M}$ genistein (asterisks) and no substrate added (filled circles)

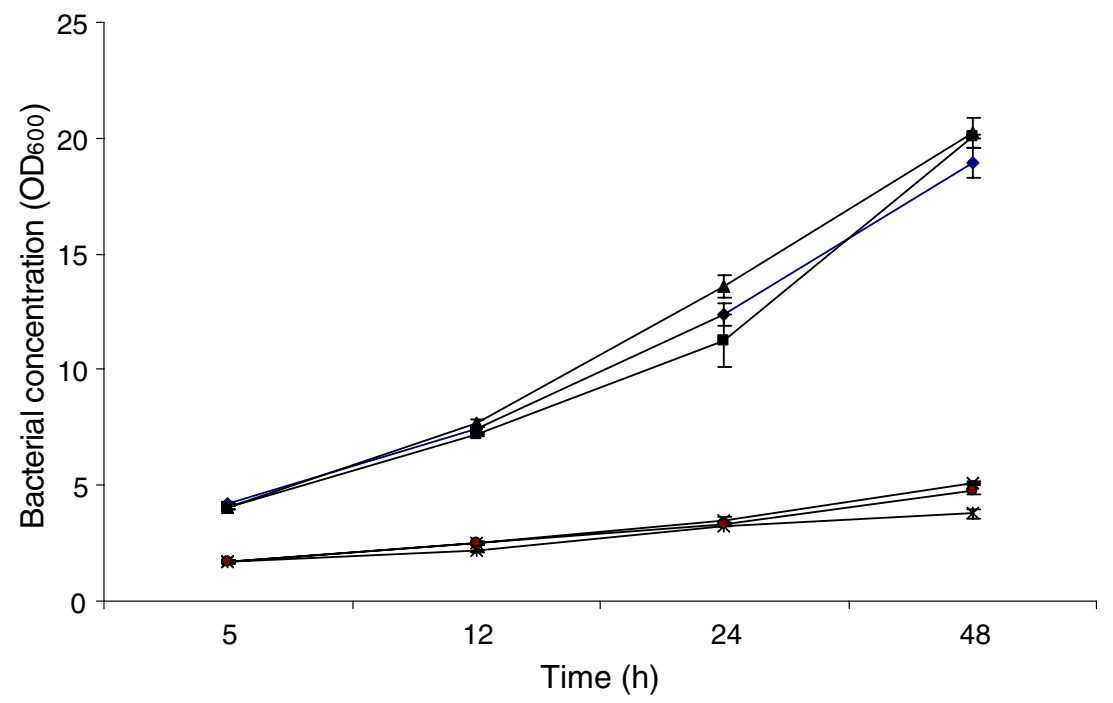

glucoside was observed in the vector control culture (Fig. S6).

Production of quercetin 3-O-glucoside in $E$. coli

Quercetin has a hydroxyl group at the 3-position of its Cring (Fig. 1), and this may be important for its anticarcinogenic activity (Ichimatsu et al. 2007; Shen et al. 2003). Glycosylation of quercetin has been reported to increase its absorption in humans (Hollman and Katan 1998, 1999; Hollman et al. 1999). Recombinant wild-type UGT71G1 produces all five potential monoglucosides from quercetin and UDP-glucose in vitro, with the B-ring 3'-O-glucoside predominating (He et al. 2006; Shao et al. 2005). However, the Phe148Val and Tyr202Ala mutants of UGT71G1 produced quercetin 3-O-glucoside as the major product in vitro ( $>95 \%$ of the total products; He et al. 2006). In the present study, feeding of quercetin to E. coli expressing Phe148Val or Tyr202Ala mutants in TB medium led to production of quercetin 3-O-glucoside as the only product

Table 3 Levels of soluble recombinant UGT proteins in E. coli cells grown with and without added substrates

\begin{tabular}{lcc}
\hline & $\mu \mathrm{g} / \mu \mathrm{L}(\mathrm{TB})$ & $\mu \mathrm{g} / \mu \mathrm{L}(\mathrm{LB})$ \\
\hline UGT71G1 & 0.48 & 0.15 \\
UGT71G1 + genistein & 0.57 & 0.19 \\
UGT71G1 + biochanin A & 0.49 & 0.15 \\
UGT71G1 + kaempferol & 0.45 & 0.13 \\
Mutant Y202A & 0.43 & 0.17 \\
Mutant Y202A + quercetin & 0.40 & 0.14 \\
Mutant Y202A + kaempferol & 0.46 & 0.19 \\
UGT73C8 & 0.59 & 0.21 \\
UGT73C8 + luteolin & 0.56 & 0.20 \\
\hline
\end{tabular}

The final concentration of substrate is $100 \mu \mathrm{M}$. The soluble protein was purified from TB and LB medium, respectively. detected. About 8- and 17-mg/L culture medium of quercetin 3-O-glucoside was produced from the cultures expressing the Phe148Val mutant when incubated with $50 \mu \mathrm{M}(16.91 \mathrm{mg} / \mathrm{L})$ and $100 \mu \mathrm{M}(33.82 \mathrm{mg} / \mathrm{L})$ quercetin, respectively, for $48 \mathrm{~h}$, while production was 10 - and $20-\mathrm{mg} / \mathrm{L}$ culture with the Tyr202Ala mutant, respectively (Fig. S7, Table 1). The conversion rate from aglycone to glucoside was about $50 \%$ after $48 \mathrm{~h}$. In comparison to LB medium, TB medium supported two to threefold higher glycoside production.

Production of kaempferol 4'-O- and 3-O-glucosides

Kaempferol is a common antioxidant in vegetables and fruits. Compared to quercetin, it contains only a single hydroxyl group (at 4') on its B-ring (Fig. 1). Kaempferol was converted to a mixture of two monoglucosides, the 4'$O$-glucoside and 3-O-glucoside, when fed to $E$. coli expressing wild-type UGT71G1, with yields of about

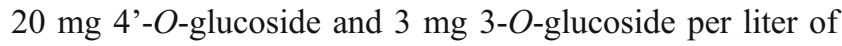
TB culture medium, respectively. However, a single product, the 3-O-glucoside, was formed when kaempferol was fed to cell cultures expressing the Phe148Val or Tyr202Ala mutants, with yields of about $16-\mathrm{mg} / \mathrm{L}$ TB culture medium (Fig. S8, Table 1).

\section{Discussion}

The isoflavone genistein and biochanin A, the flavone luteolin, and the flavonols quercetin and kaempferol have beneficial health effects for humans with regards to the prevention of cancer and cardiovascular diseases and antiinflammatory properties. Glycosylation of these compounds may significantly impact their solubility, absorption, and biological activity. This study was designed to evaluate the 
regioselectivity and effectiveness of the plant glycosyltransferases UGT73C8, UGT71G1, and mutants thereof for production of (iso)flavonoid glycosides in vivo and thus to explore the utilization of the enzymes as regioselective glycosylation biocatalysts. Genistein, biochanin A, luteolin, quercetin, and kaempferol were useful substrates for assessing regioselectivity because of the presence of multiple hydroxyl groups on these compounds. Furthermore, there is a need for production of various glycosides of these compounds to evaluate in tests with various animal disease models.

Our results showed that E. coli expressing UGT73C8, UGT71G1, or mutants of UGT71G1 collectively can regioselectively glycosylate genistein, biochanin A, luteolin, quercetin, and kaempferol to produce a specific set of glycosides in vivo. Regioselective glycosylation of these compounds by chemical approaches requires sequential blocking and deblocking of the hydroxyl groups. For example, chemical synthesis of quercetin 3-O-glucoside first required the selective protection of the catechol hydroxyl groups then glycosylation of the 3-hydroxyl group followed by deblocking of the catechol hydroxyl groups. The procedure gave a 54\% yield with contamination from product glycosylated on the 7-hydroxyl group (Bouktaib et al. 2002). On the other hand, direct isolation of significant amounts of specific flavonoid glycosides from plant sources is time-consuming since plants contain wide and variable spectra of glycosides with different types of sugar attachment (Harborne and Baxter 1999).

The enzyme system we describe here allows us to overcome many of the problems associated with efficient production of specific glucosides. Importantly, the glycosides are released to the medium, facilitating their isolation. Given that commercially available flavonoid glycosides are currently at least ten times more expensive than their corresponding aglycones expressing regioselective glycosyltransferases in E. coli in vivo is an economically feasible approach to the production of glycosides. The conversion rates in small-batch scale were from $50 \%$ to $80 \%$. However, in larger-scale production $(500-\mathrm{mL}$ cultures, ) the efficiency was reduced to $80 \%$ of the small-batch scale. Clearly, further optimization of the conditions will be needed for industry-scale production to increase the product yield.

Nucleotide-activated sugar is the essential component in small molecule glycosylation. One of the major problems for large-scale application of glycosylation is the provision of UDP-glucose to the in vitro system. Although different approaches including chemical methods (Kretzschmar and Stahl 1998), enzymatic synthesis (Bulter and Elling 1999), and regeneration using UDP-glucose pyrophosphorylase and pyrophosphatase for synthesis of nucleoside diphosphate sugars (Heidlas et al. 1992; Ichikawa et al. 1992; Wong et al. 1992) have been reported, these approaches are either laborious, difficult, or require sugar phosphates, phosphoenolpyruvate, and nucleotide 5'-triphosphate and are therefore of high cost. Since UDP-glucose is a natural intermediate in cell wall synthesis in bacterial cells, using glycosyltransferases engineered in bacteria to synthesize small molecule glycosides should be an efficient approach to overcome the difficulty associated with the preparation of nucleotide sugars.

Similar studies on production of flavonoid glycosides in $E$. coli have recently been reported (Lim et al. 2004; Willits et al. 2004). E. coli expressing UGT73B2 from Arabidopsis, with $24 \%$ sequence identity to UGT71G1, produced primarily quercetin 7-O-glucoside from quercetin, along with the 3,7-Odiglucoside as a minor product. The conversion rate was 25 $40 \%$ after 15 -h incubation with the substrate (Willits et al. 2004). Several additional UGTs from Arabidopsis, with amino acid identities to UGT71G1 from $17 \%$ to $46 \%$, also converted quercetin aglycone to different glycosides in $E$. coli. Mono or diglucosides were produced with yields from 0.19- to $10.90-\mathrm{mg} / \mathrm{L}$ culture (Lim et al. 2004). In our study, $17-20 \mathrm{mg} / \mathrm{L}$ quercetin monoglucoside was obtained. The glycosides produced in the cell culture system were also reported to release to the medium (Lim et al. 2004; Willits et al. 2004). Although the mechanisms of aglycone uptake and glycoside secretion by the bacteria are not known, several efflux pump systems have been reported suggesting that the uptake and secretion of small molecules are active processing (Kruse et al. 2002; Livshits et al. 2003; Van Dyk et al. 2004; Zakataeva et al. 1999). DMSO have been commonly used as a solvent of flavonoid substrates in enzyme activity assay in vitro or in cell culture feeding studies in vivo. Our studies showed that the bacteria continued to grow to high density during the time course and the soluble enzyme were well expressed suggesting that the DMSO concentration we used $(0.05 \%)$ did not have significant toxicity to the cells.

In the present paper, we extended our study to evaluate the utilization of two structure-directed mutants of UGT71G1 for glycosylation activity in E. coli. The Tyr202Ala mutant produced higher amounts of both quercetin and kaempferol glucosides than did the Phe148Val mutant. Amino acids Phe148 and Tyr202 are located at one end of the acceptor binding pocket and are close to each other in the threedimensional structure of UGT71G1 (Shao et al. 2005). These mutations would reduce the size of the amino acid and, in turn, increase the volume of the binding pocket and allow the 3-hydroxyl of the substrate to move closer to the C1 reaction center on the UDP-glucose for favorable glycosylation. Mutations of Phe148 to valine or Tyr202 to alanine change the regiospecificity for quercetin glycosylation from predominantly 3 '-OH to $3-\mathrm{OH}$ ( $\mathrm{He}$ et al. 2006). Our study showed that these structure-directed mutants can be used in vivo in $E$. coli to regioselectively synthesize small molecule glucosides. Advances in our understanding 
of structure-activity relationships for plant small molecule glycosyltransferases will provide more opportunities for the design of novel catalysts to produce bioactive glycosides.

Acknowledgements We thank Dr. L. Modolo for assistance with artwork and Drs Y. Z. Pang and G. J. Peel for critical reading of the manuscript. This work was supported by the Samuel Roberts Noble Foundation.

Open Access This article is distributed under the terms of the Creative Commons Attribution Noncommercial License which permits any noncommercial use, distribution, and reproduction in any medium, provided the original author(s) and source are credited.

\section{References}

Achnine L, Huhman DV, Farag MA, Sumner L, Blount JW, Dixon RA (2005) Genomic-based selection and functional characterization of triterpene glycosyltransferases from the model legume Medicago truncatula. Plant J 41:875-887

Arai Y, Watanabe S, Kimira M, Shimoi K, Mochizuki R, Kinae N (2000) Dietary intakes of flavonols, flavones and isoflavones by Japanese women and the inverse correction between quercetin intake and plasma LDL cholesterol concentration. J Nutr 130:2243-2250

Backhouse N, Rosales L, Apablaza C, Goity L, Erazo S, Negrete R, Theodoluz C, Rodriguez J, Delporte C (2007) Analgesic, antiinflammatory and antioxidant properties of Buddleja globosa, Buddlejaceae. J Ethnopharmacol 116:263-269 Nov 22 (Epub ahead of print)

Bouktaib M, Atmani A, Rolando C (2002) Regio- and stereoselective synthesis of the major metabolites of quercetin, quercetin-3-O-bD-glucuronide. Tetrahedron Lett 43:6263-6266

Bulter T, Elling L (1999) Enzymatic synthesis of nucleotide sugar. Glycoconj J 16:47-159

Caltagirone S, Rossi C, Poggi A, Ranelletti FO, Natali PG, Brunetti M, Aiello FB, Piantelli M (2000) Flavonoids apigenin and quercetin inhibit melanoma growth and metastatic potential. Int J Cancer 87:595-600

Cermak R, Landgrafs S, Wolffram S (2004) Quercetin glucosides inhibit glucose uptake into brush-border-membrane vesicles of porcine jejunum. Br J Nutr 91:849-855

Chen CY, Peng WH, Tsai KD, Hsu SL (2007) Luteolin suppresses inflammation-associated gene expression by blocking NF-kappaB and AP-1 activation pathway in mouse alveolar macrophages. Life Sci 81:1602-1614

Cogolludo A, Frazziano G, Briones AM, Cobeno L, Moreno L, Lodi F, Salaices M, Tamargo J, Perez-Vizcaino F (2007) The dietary flavonoid quercetin activates $\mathrm{BKCa}$ currents in coronary arteries via production of $\mathrm{H} 2 \mathrm{O} 2$ : Role in vasodilatation. Cardiovasc Res 73:424-431

Crespy V, Morand C, Besson C, Manach C, Demigne C, Remesy C (2001) Comparison of the intestinal absorption of quercetin, phloretin and their glucosides in rats. J Nutr 131:2109-2114

Day AJ, Gee JM, DuPont MS, Johnson IT, Williamson G (2003) Absorption of quercetin-3-glucoside and quercetin-4c-glycoside in the rat small intestine: the role of lactase phlorizin hydrolase and the sodium-dependent glucose transporter. Biochem Pharmacol 65:1199-1206

Deavours BE, Dixon RA (2005) Metabolic engineering of isoflavonoid biosynthesis in alfalfa. Plant Physiol 138:2245-2259

Erdman JW, Balentine D, Arab L, Beecher G, Dwyer JT, Folts J, Harnly J, Hollman P, Keen CL, Mazza M, Scalbert A, Vita J,
Williamson G, Burrowes J (2007) Flavonoids and heart health. J Nutr 137:718S-737S

Gabor M (1979) Anti-inflammatory substances of plant origin. In: Vane JR, Ferreira SH (eds) Handbook of experimental pharmacology: anti-inflammatory drugs. Springer, New York, pp 698-739

Gee JM, DuPont MS, Day AJ, Plumb GW, Willliamson G, Johnson IT (2000) Intestinal transport of quercetin glycosides in rats involves both deglycosylation and interaction with the hexose transport pathway. J Nutr 130:2765-2771

Graefe EU, Wittig J, Mueller S, Riethling AK, Uehmleke B, Drewelow B, Pforte H, Jacobasch G, Derendorf H, Veit M (2001) Pharmacokinetics and bioavailability of quercetin glycosides in humans. J Clin Pharmacol 41:492-499

Harborne JB, Baxter H (1999) The handbook of natural flavonoids. Wiley, New York

He XZ, Wang XQ, Dixon RA (2006) Mutational analysis of the Medicago glycosyltransferase UGT71G1 reveals residues that control regioselectivity for (iso)flavonoid glycosylation. J Biol Chem 281:3441-3447

Heidlas JE, Lees WJ, Whitesides GM (1992) Practical enzyme-based syntheses of uridine 5'-diphosphogalactose and uridine 5'diphospho- $N$-acetylgalactosamine on a gram scale. J Org Chem 57:152-157

Hertog MGL, Feskenes EJM, Hollman PC, Katan MB, Kromhout D (1993) Dietary antioxidant flavonoids and risk of coronary heart disease: the Zutphen elderly study. Lancet 342:1007-1011

Hollman PC, Katan MB (1998) Bioavailability and health effects of dietary flavonols in man. Arch Toxicol Suppl 20:237-248

Hollman PC, Katan MB (1999) Health effects and bioavailability of dietary flavonols. Free Radic Res 31(Suppl):S75-S80

Hollman PC, de Vries J, van Leeuwen SD, Mengelere MJ, Katan MB (1995) Absorption of dietary quercetin glycosides and quercetin in healthy ileostomy volunteers. Am J Clin Nutr 62:1276-1282

Hollman PC, van der Gaag M, Mengelere MJ, van Trijp J, de Vries J, Katan MB (1996) Absorption and disposition kinetics of the dietary antioxidant quercetin in man. Free Radic Biol Med 21:703-707

Hollman PC, Buysman MP, van Gameren Y, Conossen E, de Vries J, Katan MB (1999) The sugar moiety is a major determinant of the absorption of dietary flavonoid glycosides in man. Free Radic Res 31:569-573

Ichikawa Y, Lin YC, Dumas DP, Shen GJ, Garcia-Junceda E, Willams MA, Bayer R, Ketcham C, Walker LE, Paulson JC, Wong CH (1992) Chemical-enzymatic synthesis and conformational analysis of sialyl Lewis x derivatives. J Am Chem Soc 114:9283-9298

Ichimatsu D, Nomura M, Nakamura S, Moritani S, Yokogawa K, Kobayashi S, Nishioka T, Miyamoto KI (2007) Structure-activity relationship of flavonoids for inhibition of epidermal growth factor-induced transformation of JB6 CI 41 cells. Mol Carcinog 46:436-445 Jan 11 (E published)

Kao YC, Zhou C, Sherman M, Laughton CA, Chen S (1998) Molecular basis of the inhibition of human aromatase (estrogen synthetase) by flavone and isoflavone phytoestrogens: a site directed mutagenesis study. Environ Health Perspect 106:85-92

Karrasch T, Kim JS, Jang BI, Jobin C (2007) The flavonoid luteolin worsens chemical-induced colitis in NF-kappaB(EGFP) transgenic mice through blockage of NF-kappaB-dependent protective molecules. PLoS ONE 2:e596

Kellis JT, Vickerry LE (1984) Inhibition of human estrogen synthetase (aromatase) by flavones. Science 225:1032-1034

Kim JS, Jobin C (2005) The flavonoid luteolin prevents lipopolysaccharideinduced NF-kappaB signalling and gene expression by blocking lkappaB kinase activity in intestinal epithelial cells and bone-marrow derived dendritic cells. Immunology 115:375-378

Kretzschmar G, Stahl W (1998) Large-scale synthesis of linkermodified sialyl Lewis $\mathrm{X}$, Lewis $\mathrm{X}$ and $N$-acetyllactosamine. Tetrahedron 54:6341-6358 
Kruse D, Kramer R, Eggeling L, Rieping M, Pfefferle W, Tchieu JH, Chung YJ, Saier MH, Burkovski A (2002) Influence of threonine exporters on threonine production in Escherichia coli. Appl Microbiol Biotechnol 59:205-210

Kumazawa Y, Kawaguchi K, Takimoto H (2006) Immunomodulating effects of flavonoids on acute and chronic inflammatory responses caused by tumor necrosis factor alpha. Curr Pharm Des 12:4271-4279

Leonard E, Chemer J, Lim KH, Koffase MAG (2005) Expression of a soluble flavone synthase allows the biosynthesis of phytoestrogen derivatives in Escherichia coli. Appl Microbiol Biotechnol 15:1-7

Leonard E, Yan Y, Koffas MAG (2006) Functional expression of a P450 flavonoid hydroxylase for the biosynthesis of plant specific hydroxylated flavonols in Escherichia coli. Metabolic Eng 8:172-181

Lewis DA (1989) Anti-inflammatory drugs from plant and marine sources. Birkhauser, Basel

Li M, Han X, Yu B (2002) Synthesis of quercetin 3-O-(2"-galloyl)- $\alpha$ L-arabinopyranoside. Tetrahedron Lett 43:9467-9470

Lim EK, Ashford DA, Hou BK, Jackson RG, Bowles DJ (2004) Arabidopsis glycosyltransferases as biocatalysts in fermentation for regioselective synthesis of diverse quercetin glucosides. Biotechnol Bioeng 87:623-631

Liu CJ, Blount JW, Steele CL, Dixon RA (2002) Bottlenecks for metabolic engineering of isoflavone glycoconjugates in Arabidopsis. Proc Natl Acad Sci USA 99:14578-14583

Livshits VA, Zakataeva NP, Aleshin VV, Vitushkina MV (2003) Identification and characterization of the new gene $r h t A$ involved in threonine and homoserine efflux in Escherichia coli. Res Microbiol 154:123-135

Mink PJ, Scrafford CG, Barraj LM, Harnack L, Hong CP, Nettleton JA, Jacobs DR (2007) Flavonoid intake and cardiovascular disease mortality: a prospective study in postmenopausal women. Am J Clin Nutr 85:895-909

Modolo LV, Blount JW, Achnine L, Naoumkina MA, Wang XQ, Dixon RA (2007) A functional genomics approach to (iso)flavonoid glycosylation in the model legume Medicago truncatula. Plant Mol Biol 64:499-518

Shao H, He XZ, Achnine L, Blount JW, Dixon RA, Wang XQ (2005) Crystal structures of a multifunctional triterpene/flavonoid glyco- syltransferase from Medicago truncatula. Plane Cell 17:31413154

Shen SC, Ko CH, Hsu KC, Chen YC (2003) 3-OH flavone inhibition of epidermal growth factor-induced proliferation through blocking prostaglandin E2 production. Int J Cancer 108:502510

Shenouda NS, Zhou C, Browning JD, Ansell PJ, Sakla MS, Lubahn DB, MacDonald RS (2004) Phytoestrogens in common herbs regulate prostate cancer cell growth in vitro. Nutr Cancer 49:200-208

Smith GJ, Thomsen SJ, Markham KR, Andary C, Cardon D (2000) The photo stabilities of naturally occurring 5-hydroflavones, flavonols, their glycosides and their aluminium complexes. J Photochem Photobiol 136:87-91

Van Dyk TK, Templeton LJ, Cantera KA, Sharpe PL, Sariaslani FS (2004) Characterization of the Escherichia coli AaeAB efflux pump: a metabolic relief valve? J Bacteriol 186:7196-7204

Way TD, Kao MC, Lin JK (2004) Apigenin induces apoptosis through proteasomal degradation of HER2/neu in HER2 neu-overexpressing breast cancer cells via the phosphatidylinositol 3-kinase Aktdependent pathway. J Biol Chem 279:4479-4489

Willits MG, Giovanni M, Orata RTN, Kramer CM, Luca VD, Steffens JC, Graser G (2004) Bio-fermentation of modified flavonoids: an example of in vivo diversification of secondary metabolites. Phytochem 65:31-41

Wong CH, Wang R, Ichikawa Y (1992) Regeneration of sugar nucleotide for enzymatic oligosaccharide synthesis: use of Gal-1phosphate uridyltransferase in the regeneration of UDP-galactose, UDP-2-deoxygalactose, and UDP-galactosamine. J Org Chem $57: 4343-4344$

Yu O, Shi J, Croes RA, Fader GM, McGonigle B, Odell JT (2000) Production of the isoflavones genistein and daidzein in nonlegume dicot and monocot tissues. Plant Physiol 124:781-794

Yu O, Shi J, Hession AO, Maxwell CA, McGonigle B, Odell JT (2003) Metabolic engineering to increase isoflavone biosynthesis in soybean seed. Phytochem 63:73-76

Zakataeva NP, Aleshin VV, Tokmakova IL, Troshin PV, Livshits VA (1999) The novel transmembrane Escherichia coli proteins involved in the amino acid efflux. FEBS Lett 452:228-232 